\title{
Haematology
}

\section{PRIMARY PLASMA CELL LEUKAEMIA WITH MULTIPLE EXTRAMEDULLARY INVOLVEMENT}

Kwok-Hei Chan $^{1}$, Grace W. N. Lau ${ }^{2}$, Harold K. K. Lee ${ }^{2}$, Kate F. S. Leung

${ }^{1}$ Department of Pathology, Princess Margaret Hospital, Kowloon, Hong Kong; and ${ }^{2}$ Department of Medicine and Geriatrics, Princess Margaret Hospital, Kowloon, Hong Kong

A 51-year-old man presented with left facial swelling and a gingival mass originating from left maxilla. He developed progressive right proptosis with visual loss and subsequently passed melena.

Laboratory tests showed leucocytosis $\left(27.8 \times 10^{9} / \mathrm{L}\right)$ with $32 \%$ circulating plasmablasts $\left(8.9 \times 10^{9} / \mathrm{L}\right)$, anaemia $(\mathrm{Hb} 95 \mathrm{~g} / \mathrm{L})$, acute kidney injury (creatinine $149 \mathrm{mmol} / \mathrm{L})$, hypercalcaemia $(2.86$ $\mathrm{mmol} / \mathrm{L}$ ) and IgG-lambda paraproteinaemia (40 g/L). Flow cytometry confirmed lambda light chain restriction of the plasmablasts $\left(\mathrm{CD} 138^{+} \mathrm{CD} 10^{-} \mathrm{CD}^{+} 6^{+}\right)$. CT scan identified soft tissue masses in left maxillary antrum and right orbit with optic nerve compression. Oesophago-gastroduodenoscopy found multiple ulcerated nodules in stomach. Gingival and gastric biopsies showed high-grade haematolymphoid malignancy with plasmacytic differentiation. Bone marrow examination revealed infiltration by sheets of plasmablasts which were negative for EBER. Anti-HIV serology was negative. Cytogenetic study reported a complex karyotype while FISH demonstrated gain of CKS1B and deletion of TP53. These findings are consistent with primary plasma cell leukaemia (PCL). The patient achieved very good partial response with VTD-PACE chemotherapy and was intended for autologous haematopoietic stem cell transplantation. However, he developed frank relapse before stem cell harvest and eventually succumbed despite salvage therapy.

This case highlights the highly variable manifestation of PCL and underscores the importance of multidisciplinary inputs towards prompt and accurate diagnosis.

\section{GAUCHER DISEASE PRESENTING AS LONGSTANDING SPLENOMEGALY AND THROMBOCYTOPENIA - A CASE REPORT}

Sonia Raj, Roya Arabi, Sam Hitchins, Archna Sharma, Anna Hutchinson

Department of Haematology, Royal Hobart Hospital, Tas, Australia

Gaucher disease is an autosomal dominant inborn error of metabolism that affects the recycling of cellular glycolipids and is a deficiency of a lysosomal enzyme glucocerebrosidase. We report a case of a 26-year-old lady who had long standing, unexplained isolated splenomegaly. This is on a background of four prior pregnancies during which she was noted to have gestational thrombocytopenia. She was first diagnosed with splenomegaly three years ago with a spleen size of $19 \mathrm{~cm}$ with intermittent splenic pain exacerbated by exercise and alcohol intake. She was observed initially as these were around the times of her pregnancies. Following the birth of her fourth child, she underwent a PET scan to assess for evidence of splenic lymphoma and this revealed an enlarged spleen with normal FDG uptake and no FDG uptake elsewhere. Prior to the decision of a splenic biopsy, a bone marrow biopsy was undertaken which was suggestive of a lysosomal storage disorder, in particular that of Gaucher disease. This was then confirmed via enzyme analysis of glucocerebrosidase activity and targeted DNA analysis. Mutation analysis revealed a GBA:c.1584T $>\mathrm{G}$ variant which is of uncertain significance and not known to be an associated variant in Gaucher disease.

\section{Reference}

1. Thomas AS, Mehta AB, Hughes DA. Diagnosing Gaucher disease: an on-going need for increased awareness amongst haematologists. Blood Cells Mol Dis 2013; 50: 212.

\section{AN UNEXPECTED CAUSE OF PSEUDO-PELGER- HUET ANOMALY}

Jeremy Ong, Radha Ramanan, Jay Hocking, Sue Morgan Alfred Health, Melbourne, Vic, Australia

Pseudo-Pelger-Huët anomaly (PPHA) is an acquired disorder of neutrophil morphology resembling the inherited condition, Pelger-Huët anomaly. We report a case of PPHA apparently caused by acute parvovirus infection.

A 51-year-old woman presented with fevers and cytopenias (mild neutropenia $1.4 \times 10^{9} / \mathrm{L}$, moderate thrombocytopenia $\left.74 \times 10^{9} / \mathrm{L}\right)$. Her history was significant for hypothyroidism treated with thyroxine. Her only other medication was the oral contraceptive pill. Peripheral blood morphology showed many hyposegmented neutrophils with abnormal nuclear chromatin clumping. Red cell and platelet morphology was unremarkable. A blood film from the previous year showed normal neutrophil morphology.

Based on a presumptive diagnosis of myelodysplastic syndrome (MDS), a bone marrow examination was performed. It demonstrated a mildly hypocellular marrow with adequate granulopoiesis, frequent Pelger-Huët cells and no increase in blasts. Megakaryocyte number and morphology were normal. Unexpectedly, erythropoiesis was markedly reduced consistent with pure red cell aplasia (PRCA). Concurrent reticulocytopenia was observed.

Subsequent testing showed positive $\operatorname{IgM}$ and $\operatorname{IgG}$ parvovirus serology, consistent with recent parvovirus infection. Our patient's cytopenias spontaneously resolved within a week. Four months after initial presentation, full blood parameters remain within the reference range, though Pelger-Huët cells persist. PPHA is seen with clonal haematological disorders (MDS, myeloproliferative neoplasms), certain medications ${ }^{1}$ (tacrolimus, mycophenolate, valproate, ganciclovir, docetaxel and ibuprofen) and infections ${ }^{2}$ (HIV, tuberculosis and mycoplasma). Our case of PPHA likely resulted from parvovirus infection, which was only incidentally discovered after a bone marrow biopsy showed 